SHORT REPORT

\title{
Brain stem and cerebellar dysfunction after lumbar spinal fluid drainage: case report
}

\author{
J Bloch, L Regli
}

J Neurol Neurosurg Psychiatry 2003;74:992-994

Lumbar spinal fluid drainage is a common procedure to reduce the risks of cerebrospinal fluid (CSF) fistula after skull base fractures or various transdural neurosurgical procedures. Nevertheless, this simple and effective technique can lead to overdrainage and CSF hypovolaemia. This report describes the case of a young patient who had a lumbar drain inserted, to avoid CSF fistula after a pterional craniotomy with opening of the frontal sinus for the clipping of a ruptured aneurysm. The drain was removed after 48 hours because of underdrainage $\quad<1$ $\mathrm{ml} / \mathrm{h}$ ). Three days after drain removal, she developed rapid deterioration of her level of consciousness and signs of cranial nerves involvement, brain stem and cerebellar dysfunction. Intracranial pressure was low $\left(<5 \mathrm{~cm} \mathrm{H}_{2} \mathrm{O}\right)$ and MRI showed brain sagging and cerebellar foramen magnum herniation. The patient was successfully treated with epidural blood patch, ventricular drainage, and Trendelenburg position. The authors report this case because CSF hypovolaemia attributable to lumbar overdrainage is an insidious and threatening condition not easy to diagnose in the absence of detectable CSF leak. MRI and intracranial pressure monitoring confirm the diagnosis and permit better understanding of the physiopathology of brain sagging.

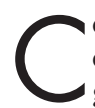
erebrospinal fluid (CSF) leakage is a classic neurosurgical complication after cerebral or spinal transdural surgery or after fracture of the skull base. ${ }^{1}$ Continuous CSF lumbar drainage has gained wide acceptance to prevent and treat such fistulas, as it decreases the pressure gradient across the fistula, and offers an attractive alternative to surgical revision that exposes the patient to anaesthetic and procedural risks. ${ }^{23}$ One has, however, to be aware of the risks related to this simple and effective procedure. To illustrate the severe complications that can be associated with lumbar drainage, we present a case of brain stem and cerebellar dysfunction attributable to CSF hypovolaemia that developed several days after a lumbar drain had been removed. We discuss the concept of CSF hypovolaemia and propose therapeutical strategies.

\section{CASE REPORT}

A 38 year old healthy woman was admitted for a Fisher grade II subarachnoid haemorrhage attributable to a ruptured carotid artery bifurcation aneurysm, without hydrocephalus. Neurological examination was normal except for meningeal signs.

A pterional craniotomy was performed to clip the aneurysm. Because of extensive pneumatisation of the frontal skull base, the frontal sinus was opened during craniotomy. To prevent the development of a CSF fistula, the frontal sinus was cranialised and a lumbar drainage was inserted. Two puncture

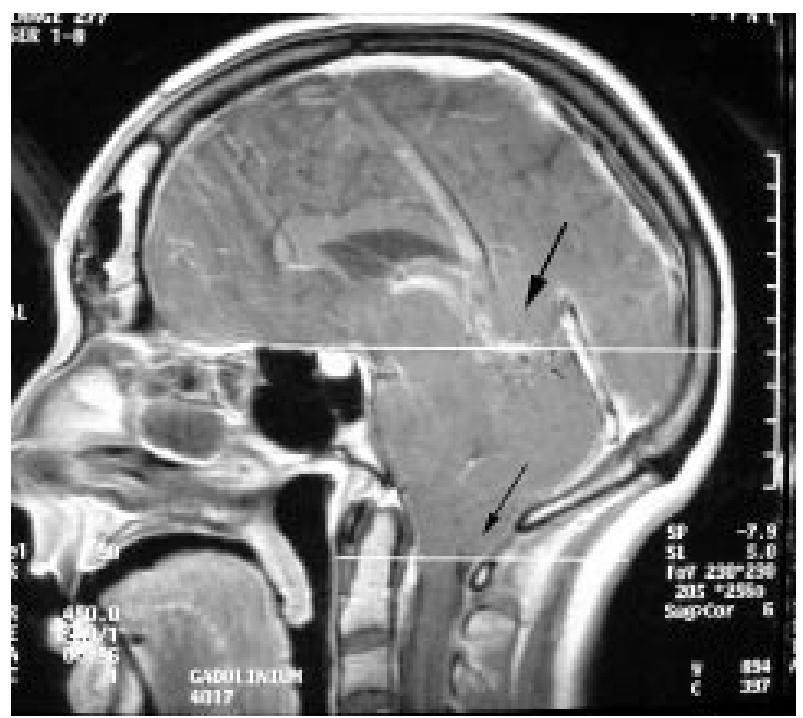

Figure $1 \mathrm{Tl}$ gadolinium enhanced mid-sagittal cerebral MRI shows the sagittal sinus without meningeal enhancement. The large arrow shows the tentorial herniation of the splenium of the corpus callosum and the cingular gyrus. The transtentorial herniation reaches a line drawn tangential to the frontal cranial base. The thin arrow shows the tonsillar herniation through the foramen magnum. The tip of the tonsils descends to the upper part of the arch of $\mathrm{Cl}$ as indicated by the lower line (drawn parallel to the upper line). Note also the disappearance of the posterior fossa cisterns and the fourth ventricle.

attempts with an 18 gauge needle were needed to place the drain intradurally.

The patient had normal postoperative neurological examination. Drainage through the lumbar catheter was minimal $(<1 \mathrm{ml} / \mathrm{h})$, for this reason it was removed the second postoperative day. Three days after drain removal, she developed headache and drowsiness. On examination, she had difficulties swallowing, absent gag reflex, VIth nerve palsy on the left side, and multidirectional nystagmus.

Cerebral computed tomography showed small lateral ventricles, grey-white matter dedifferentiation in the posterior fossa, and descent of the cerebellar tonsils into the occipital foramen. Cerebral angiogram showed complete exclusion of the aneurysm as well as the absence of cerebral vasospasm.

Intracranial hypotension was in the differential diagnosis, however there was no clinical evidence of CSF leakage. Cerebral MRI (fig 1) confirmed the presence of tonsillar herniation through the foramen magnum and tentorial herniation of the splenium of the corpus callosum as well as of the cingular gyrus without pachy-meningeal gadolinium enhancement, cerebral oedema, venous obstruction, or ischaemia.

By that time, the patient was lethargic and was intubated. We decided to monitor her intracranial pressure and to perform an external ventricular drainage. The intracranial 


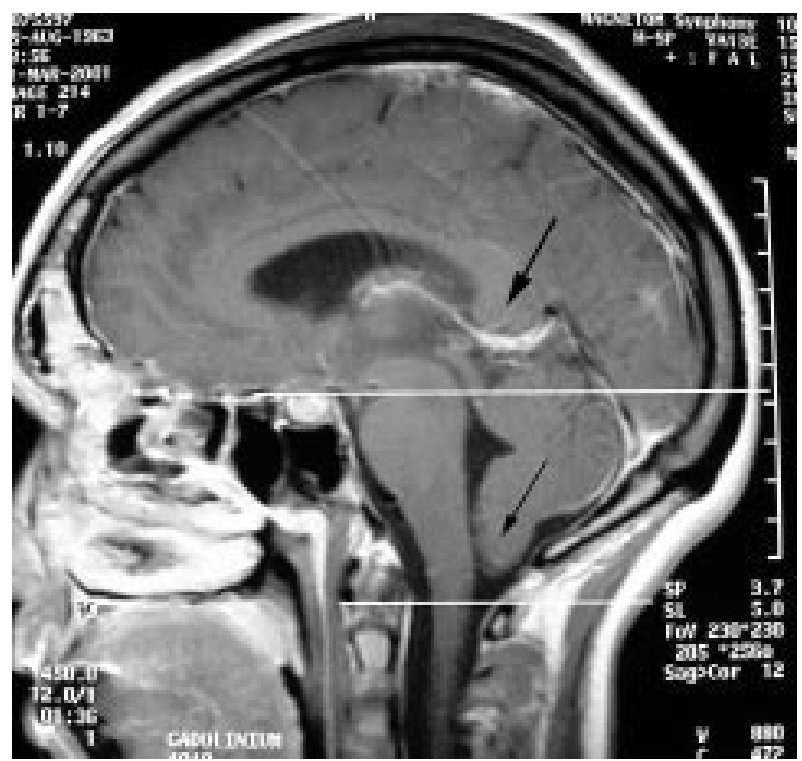

Figure 2 Tl gadolinium enhanced mid-sagittal cerebral MRI. It shows normal anatomical relations with recovery of the transtentorial and transforamen magnum herniation. (The two drawn lines are identical to figure 1). Note also the reappearance of the perimedullar spaces are open.

pressure was low $\left(<5 \mathrm{~cm} \mathrm{H}_{2} \mathrm{O}\right.$ ). This information confirmed the diagnosis of intracranial hypotension. Even though no CSF leakage or collection was detectable in the lumbar area, the most probable cause was an occult spinal fluid fistula consecutive to the lumbar drain punctures.

To promote dural repair, we performed a lumbar epidural blood patch at the site of puncture. To help posterior fossa structures to raise back to their normal position and to decrease the pressure gradient across the fistula, we positioned her head down $10^{\circ}$ and set the external ventricular drain at $+5 \mathrm{~cm} \mathrm{H}_{2} \mathrm{O}$. This treatment combination resulted in a rapid improvement of the patient's neurological status, with full recovery in 24 hours. Five days later, control MRI (fig 2) showed complete resolution of the brain sagging and cerebellar foramen magnum herniation.

The patient was progressively allowed to be in the upright position and was discharged home with a normal neurological examination five days later.

\section{DISCUSSION}

CSF diversion has been widely used in the prevention and treatment of traumatic and iatrogenic CSF fistulas. Although it is an effective procedure, CSF overdrainage may result in serious complications. The number of puncture attempts, as well as the size and shape of the needle may increase the risk of post procedure CSF leak. Common adverse events observed with lumbar spinal fluid drainage are infections, ${ }^{4}$ neurological injury from spinal drain insertion, ${ }^{5}$ pneumocephalus, ${ }^{6}$ haematomas, and CSF overdrainage.$^{57}$ A recent study reviewing 530 consecutive lumbar drains for trans-sphenoidal surgery reports an incidence of $2.5 \%$ for postural headache related to overdrainage, only $1.3 \%$ of them required an epidural blood patch. ${ }^{7}$ Although postural headache is a minor symptom, major events can be related to overdrainage for instance, VIth cranial nerve palsy, binasal field deficit, transient visual obscuration, hyperacousia, vertigo, vocal cord paralysis, occlusion of the posterior cerebral artery because of cerebellar foramen magnum herniation, and decreased level of consciousness have been described. ${ }^{511}$ In all the cases reported in the literature except for one that died, ${ }^{5}$ alleviation of the complications attributable to overdrainage was achieved by cessation of lumbar drainage. Interestingly, our patient became symptomatic three days after removal of the lumbar drain and while she was at bed rest. Moreover, the lumbar catheter had not drained properly during the 48 hours before removal $(<1$ $\mathrm{ml} / \mathrm{h}$ ). Consequently, CSF overdrainage is an insidious abnormality that deserves attention even after the drain has been removed.

Intracranial hypotension is not only related to spinal lumbar overdrainage, ${ }^{58}$ but also to CSF leaks after trauma, spinal surgery, ${ }^{9}$ or spontaneous spinal CSF leaks as for example after rupture of Tarlov cyst. ${ }^{1011} 12$

In a recent paper, Mokri reviews the clinical and imaging features associated with the syndrome of CSF hypovolaemia. ${ }^{13}$ According to his review, there is no linear correlation between CSF volume and pressure, and the common physiopathological denominator in this syndrome is hypovolaemia and not intracranial hypotension. He classifies patients with intracranial hypovolaemia depending on the presence or not of intracranial hypotension, pachymeningeal gadolinium enhancement on imaging studies, and clinical symptoms:

- Type I, the classic form, presents with intracranial hypotension, diffuse pachymeningeal gadolinium enhancement, and clinical signs.

- Type II, equal to type I, but with a normal CSF pressure.

- Type III, equal to type I, but without pachymeningeal enhancement on imaging.

- Type IV, equal to type I, but without clinical symptoms.

MRI helps to diagnose CSF hypovolaemia, even though dural enhancement, brain sagging, and subdural collections are not invariable findings in this syndrome. ${ }^{913} 14$

The patient we present can be classified as hypovolaemia syndrome type III, as she had low intracranial pressure, no pachymeningeal gadolinium enhancement, and rapid clinical deterioration over a few hours. Such a rapid deterioration is uncommon, as most of the cases reported exhibited a much slower evolution over weeks or longer.

Although, hypovolaemia is an attractive concept to explain brain sagging, we believe that CSF dynamics contribute in a determinant manner to this phenomenon. In a simple physical model in which the brain and spinal cord would be freely floating in a non-viscous fluid, lumbar drainage or ventricular drainage would not induce brain sagging. In reality, the human model is far more complex, because many additional parameters influence CSF circulation, ${ }^{15}$ such as fluid viscosity, anatomy of the arachnoidal cisterns and paccionan granulations, bridging veins, and elasticity of the meningeal membranes. All these parameters differ from one patient to the other, because of constitutional, as well as acquired factors (for example, subarachnoid haemorrhage as in our patient). This is why we believe that patients are at higher risk to develop pressure gradients between the cranial and spinal compartment, and therefore brain sagging after lumbar drainage, than after ventricular drainage. Mokri's review on CSF hypovolaemia supports this, as brain sagging was described only in patients with spinal CSF leaks, and never in patients with cranial CSF leaks. ${ }^{13}$ The dramatic improvement obtained in our patient by combining external ventricular drainage and head down positioning further supports this hypothesis. The clinical recovery was not related to reestablishment of CSF normovolaemia (as we continued to drain CSF from the ventricle), but to the inversion of CSF dynamics between the spinal and the cranial compartments.

Treatment of spinal dural leaks is often successful after one or more epidural patches ${ }^{16}$; if this fails, surgery to repair the dural defect may be necessary. ${ }^{10}{ }^{11}$ In the presence of progressive brain sagging, symptoms may become severe and life threatening. In such situation, we recommend the addition of Trendelenburg position and external ventricular CSF drainage to invert the pressure gradient between the spinal and cranial compartment and to decrease the pressure at the level of the fistula. 
Furthermore, measurement of low intracranial pressures with the ventricular drain confirms the diagnosis and permits monitoring during the Trendelenburg positioning of a neurologically threatened patient.

In conclusion the use of lumbar spinal drainage is a procedure that deserves special attention and careful monitoring as it may be associated with life threatening CSF overdrainage or leak, even after removal of the lumbar drain. The risk of dural tear and subsequent occult CSF fistula is increased after multiple puncture attempts to insert the drain. A therapeutic strategy, combining Trendelenburg positioning and external ventricular drainage was successfully applied in one patient presenting severe life threatening brain sagging attributable to occult CSF leak.

\section{Authors' affiliations}

J Bloch, L Regli, Department of Neurosurgery, CHUV, Lausanne, Switzerland

Correspondence to: Dr L Regli, Service de Neurochirurgie, BH-13, 1011 Lausanne CHUV, Switzerland; Luca.Regli@chuv.hospvd.ch

Received 1 October 2002

Accepted in final revised form 11 January 2003

\section{REFERENCES}

1 Couldwell WT. Cerebrospinal fluid fistulas. In: Youmans JR, ed. Neurosurgery. New York: McGraw Hill, 1989:2329-42.

2 Houle PJ, Vender JR, Fountas K, et al. Pump-regulated lumbar subarachnoid drainage. Neurosurgery 2000;46:929-32.

3 Vourc'h G. Continuous cerebrospinal drainage by indwelling spinal catheter. Br J Anesth 1963;35:118-20.
4 Kitchel S, Eismont F, Green B. Closed subarachnoid drainage for management of cerebrospinal fluid leakage after an operation on the spine. J Bone Joint Surg 1989;71A:984-7.

5 Roland PS, Marple BF, Meyerhoff WL, et al. Complication of lumbar spinal fluid drainage. Otolaryngol Head Neck Surg 1992;107:564-9.

6 Graf C, Gross C, Bech D. Complications of spinal drainage in the management of cerebrospinal fluid leakage after operation on the spine. J Neurosurg 1981;34:392-5.

7 Grady RE, Horlocker TT, Brown RD, et al. Neurologic complications after placement of cerebrospinal fluid drainage catheters and needles in anesthetized patients: implications for regional anethesia. Anesth Analg 1999;88:388-92.

8 Sathi S, Stieg PE. "Acquired" Chiari I malformation after multiple lumbar puctures: case report. Neurosurgery 1993:32:306-9.

9 Schievink WI, Tourje J. Intracranial hypotension without meningeal enhancement on magnetic resonance imaging. J Neurosurg 2000;92:475-7.

10 Atkinson JLD, Weinshenker BG, Miller GM, et al. Acquired Chiari I malformation secondary to spontaneous spinal cerebrospinal fluid leakage and chronic intracranial hypotension sydrome in seven cases. $J$ Neurosurg 1998;88:237-42.

11 Pleasure SJ, Abosch A, Friedman J, et al. Spontaneous intracranial hypotension resulting in stupor caused by diencephalic compression. Neurology 1998;50: 1854-7.

12 Schievink WI, Meyer FB, Atkinson JSD, et al. Spontaneous spinal cerebrospinal fluid leaks and intracranial hypotension. J Neurosurg 1996;84:598-605.

13 Mokri B. Spontaneous cerebrospinal fluid leaks. From intracranial hypotension to cerebrospinal fluid hypovolemial-evolution of a concept. Mayo Clin Proc 1999;74:1113-23.

14 Fishman RA, Dillon WP. Dural enhancement and cerebral displacement secondary to intracranial hypotension. Neurology 1993;43:609-11.

15 Ursino $M$, Lodi CA. A simple mathematical model of the interaction between intracranial pressure and cerebral hemodynamics. J of Applied Physiol 1997;82:1256-69.

16 Rupp SM, Wilson CB. Treatment of spontaneous cerebrospinal fluid leak with epidural blood patch. J Neurosurg 1989;70:808-10.

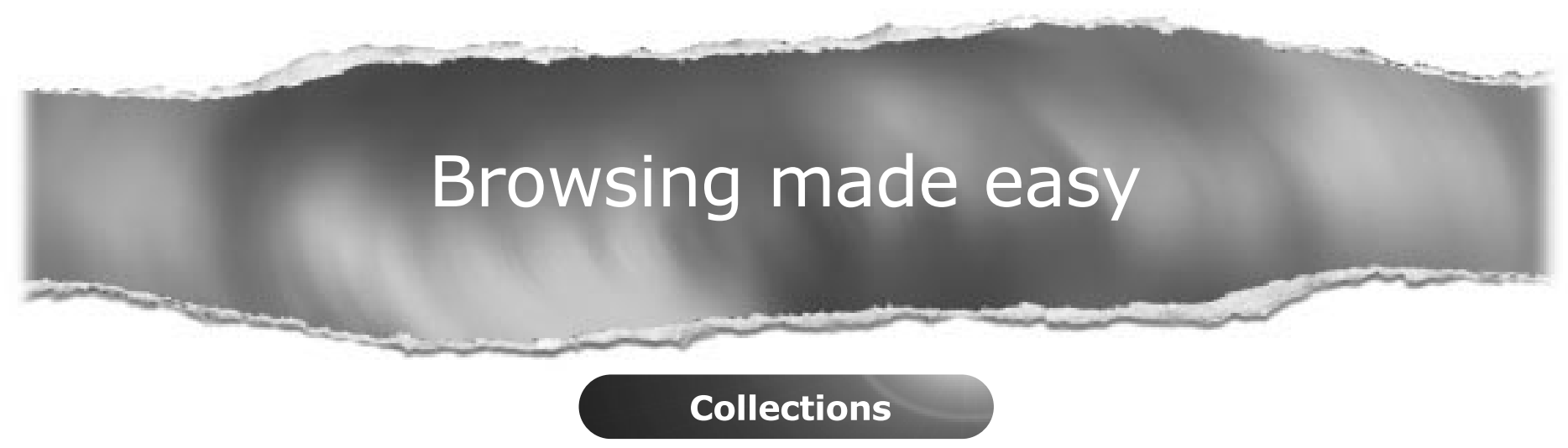

With a single click Collections allows you to find all articles that have been published in your chosen subject. Select from over 200 clinical and non-clinical topic collections and/or cross search other specialist journals, the BMJ and Cochrane Reviews

\section{www.jnnp.com}

